$01,08,11$

\title{
Модификация атомной и электронной структур палладия в результате растворения водорода
}

\author{
(С) Л.Ю. Немирович-Данченко ${ }^{1,2}$, О.В. Лопатина ${ }^{1}$, Л.А. Святкин ${ }^{1}$, И.П. Чернов ${ }^{1}$ \\ ${ }^{1}$ Национальный исследовательский Томский политехнический университет, \\ Томск, Россия \\ ${ }^{2}$ Томский государственный университет систем управления и радиоэлектроники, \\ Томск, Россия \\ E-mail: ndlyu@tpu.ru
}

Поступила в Редакцию 23 марта 2021 г.

В окончательной редакции 5 апреля 2021 г.

Принята к публикации 5 апреля 2021 г.

Представлены результаты изучения из первых принципов особенностей изменения атомной и электронной структур палладия в результате растворения водорода. Показано, что энергия связи водорода в палладии ведет себя нерегулярным образом: с увеличением концентрации водорода $x=\mathrm{H} / \mathrm{Pd}$ до 0.75 она растет, а затем падает, принимая отрицательные значения при концентрациях водорода $x$ более 1.5 . Установлено, что в результате растворения водорода в палладии наблюдается перенос заряда от атомов Рd к атомам $\mathrm{H}$. Величина этого переноса заряда растет по мере насыщения палладия водородом.

Ключевые слова: водород, палладий, энергия связи, перенос заряда, плотность электронных состояний.

DOI: 10.21883/FTT.2021.08.51147.059

\section{1. Введение}

Исследование материалов способных накапливать водород приобретает особенное значение в связи с развитием водородной энергетики. Из всех металлов, поглощающих водород, самым известным является палладий. Растворение водорода значительно изменяет физические и механические свойства палладия: увеличивает параметр решетки, снижает электропроводность и магнитную восприимчивость, повышает твердость и прочность, снижает пластичность и, как результат, приводит к охрупчиванию материала. Изучению свойств системы $\mathrm{Pd}-\mathrm{H}$ посвящены многочисленные экспериментальные и теоретические исследования [1-21], тем не менее остаются вопросы, на которые пока нет полного ответа. Так, например, неясна природа концентрационной зависимости энергии связи водорода в палладии $[10,13,14,17,19]$ и электрического сопротивления системы палладийводород [22-24], стимулированного выхода водорода излучением [25-28] и т.д. Ключ к более глубокому пониманию этих вопросов, как нам представляется, состоит в комплексном исследовании модификации атомной и электронной структур палладия при растворении в нем водорода. Исследуя атомную структуру, можно определить энергетически наиболее выгодные положения водорода в решетке палладия, проследить за изменением параметров решетки и пространственным расположением атомов $\mathrm{Pd}$ и Н. Изучение плотности электронных состояний и переноса заряда в системе $\mathrm{Pd}-\mathrm{H}$ позволит получить информацию о влиянии электронов атомов водорода на электронную структуру $\mathrm{Pd}$, на изменение количества электронов проводимости и продвинутся в понимании природы охрупчивания. Следует заметить, что подобные исследования для системы $\mathrm{Pd}-\mathrm{H}$ проводились ранее, однако, как правило, для отдельных концентраций водорода [6-9,15-17,19,21] или для объяснения отдельных вопросов, связанных с поведением водорода в палладии, например, ускоренной диффузии Н при радиационном воздействии [26-28].

Целью настоящей работы является изучение влияния концентрации водорода на атомную и электронную структуры системы палладий-водород. С этой целью были проведены расчеты из первых принципов атомной структуры, переноса заряда и плотности электронных состояний системы палладий-водород при концентрации водорода $x=\mathrm{H} / \mathrm{Pd}$ в диапазоне $0.25-2.0$.

\section{2. Метод и детали расчета}

Оптимизация параметров решетки и релаксация положений всех атомов в расчетной ячейке системы палладий-водород проводились в рамках теории функционала электронной плотности методом оптимизированного сохраняющего норму псевдопотенциала Вандербильта [29], реализованным в пакете программа ABINIT [30,31]. Обменно-корреляционные эффекты учитывались с использованием обобщенного градиентного приближения в форме Пердью-Бурке-Ернцерхофа (PBE) [32]. Энергия обрезания при разложении по базису плоских волн составляла $816 \mathrm{eV}$. В работе была исследована атомная и электронная структуры системы $\mathrm{PdH}_{x}$ при $x$ равном $0.25,0.5,0.75,1.0,1.25,1.5$, 1.75 и 2.0, в которых атомы водорода занимали либо тетра- (Т), либо октаэдрические $(\mathrm{O})$ междоузлия, а так- 

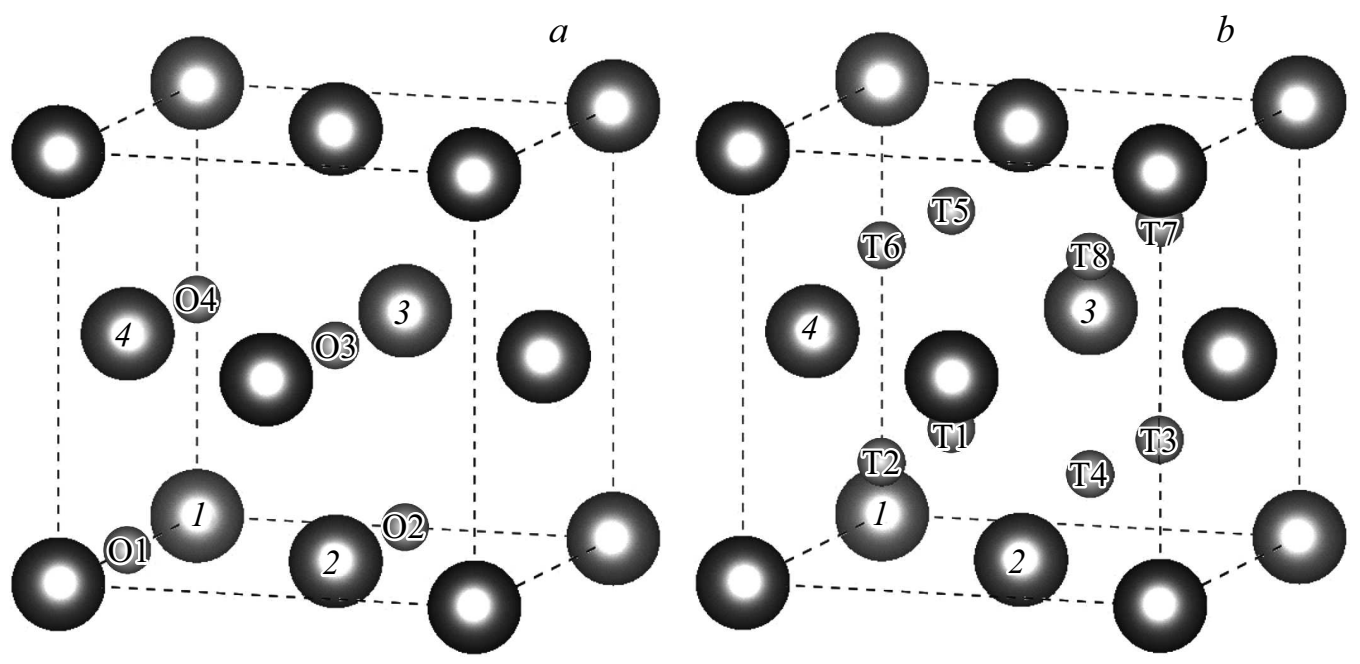

Рис. 1. Расчетные ячейки соединения $\left.\mathrm{PdH}_{x}: a\right)$ атомы водорода в октаэдрических междоузлиях, $\left.b\right)$ атомы водорода в тетраэдрических междоузлиях; большие шарики - палладий, маленькие шарики — водород.

же О и Т междоузлия одновременно (рис. 1). Расчетные ячейки состояли из 4 атомов палладия. Для удобства обсуждения атомы палладия и водорода в расчетных ячейках на рис. 1 пронумерованы. Набор $k$-точек составлял $10 \times 10 \times 10$. В нашей работе самосогласование электронной плотности считалось достигнутым, когда сходимость полной энергии расчетной ячейки составляла $\sim 0.001 \mathrm{meV}$. Для всех рассмотренных систем оптимизация параметра решетки, а также релаксация положений атомов в расчетной ячейке была проведена с использованием алгоритма Бройдена-ФлетчераГольдфарба-Шанно [33-36]. Процесс релаксации считался завершенным, когда силы, действующие на каждый атом в расчетной ячейке, становились меньше $5 \mathrm{meV} / \AA$. Плотности электронных состояний (ПЭС) рассчитаны линейным методом тетраэдронов. Радиусы МТ-сфер атомов палладия и водорода для расчета парциальных ПЭС были выбраны равными 1.217 и $0.529 \AA$, соответственно. Сглаживание ПЭС осуществлялось гауссианами со среднеквадратичной шириной $0.13 \mathrm{eV}$. Объем атомов и перенос заряда рассчитаны по методу Бадера [37].

\section{3. Концентрационная зависимость атомной структуры системы $\mathrm{PdH}_{x}$}

Результаты наших расчетов показали, что при заполнении водородом как октаэдрических, так и тетраэдрических междоузлий решетка палладия после релаксации остается ГЦК. Однако при одновременном заполнении водородом октаэдрических и тетраэдрических междоузлий (для концентраций $x$ равных 1.0-2.0) наблюдается заметное тетрагональное искажение решетки палладия. Вычисленные в настоящей работе значения параметров решетки представлены в таблице для энергетически наиболее выгодных конфигураций водорода в рассмотрен-

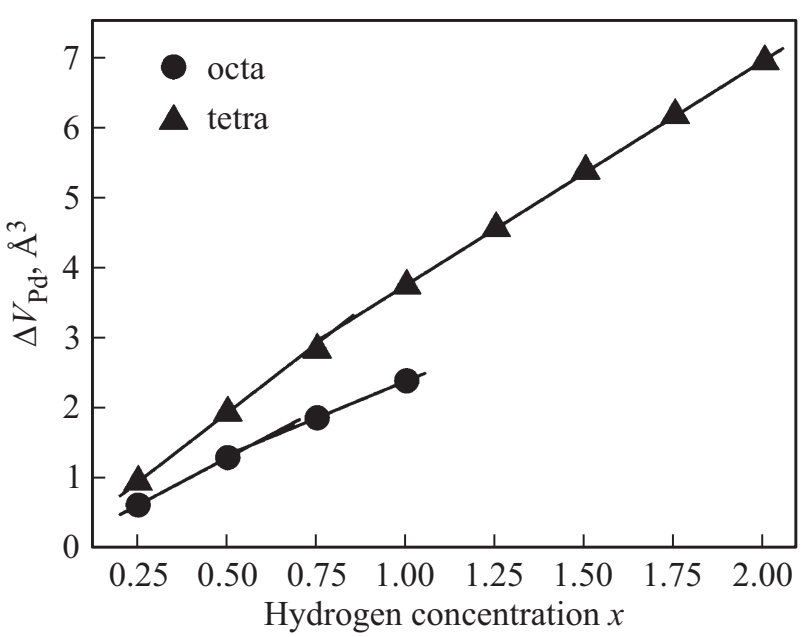

Рис. 2. Избыточный объем, вносимый атомом водорода на один атом Pd, в зависимости от концентрации $x$.

ных соединениях $\mathrm{PdH}_{x}$. Рассчитанные нами параметры решетки находятся в хорошем согласии, как с теоретическими $[9,14,15,18,21]$, так и с экспериментальными результатами $[2,6,7,38]$ других работ.

В работе рассчитан избыточный объем $\Delta V_{\mathrm{Pd}}$, вносимый атомом Н на один атом $\mathrm{Pd}$. Результаты расчета представлены на рис. 2 в зависимости от концентрации водорода $x$ в палладии. Зависимость имеет линейный характер с разными угловыми коэффициентами в диапазонах $0<s<0.5$ и $0.75<x<2.0$. Пересечение этих линий указывает на концентрацию фазового перехода, поскольку известно, что в первом диапазоне концентраций $x$ реализуется $\alpha$-фаза, а во втором - $\beta$-фаза системы палладий-водород $[1,4,11,12,22]$. Согласно нашим расчетам, фазовый переход происходят при $x_{\mathrm{ph}}=0.57$ для октаэдрической и при $x_{\mathrm{ph}}=0.80$ для тетраэдри- 
Параметры решетки для энергетически наиболее выгодных конфигураций водорода в системе РdН . (Занимаемые атомами водорода междоузлия указаны согласно обозначениям, представленным на рис. 1)

\begin{tabular}{|c|c|c|c|c|}
\hline \multirow{2}{*}{$\begin{array}{c}\text { Концентрация } \\
\text { водорода } x\end{array}$} & $a, \AA$ & $\begin{array}{l}\text { Занимаемые } \\
\text { междоузлия }\end{array}$ & $a, \AA$ & $\begin{array}{l}\text { Занимаемые } \\
\text { междоузлия }\end{array}$ \\
\hline & \multicolumn{2}{|c|}{ Водород в октаэдрическом междузлии } & \multicolumn{2}{|c|}{ Водород в тетраэдрическом междузлии } \\
\hline 0 & $\begin{array}{l}3.940 \\
3.88[18] \\
3.97[14] \\
3.92[6,9] \\
3.959[15] \\
3.93[21]\end{array}$ & - & 3.94 & \\
\hline 0.25 & $\begin{array}{l}3.995 \\
4.02[14] \\
4.018[15]\end{array}$ & $\mathrm{O} 1$ & $\begin{array}{l}4.018 \\
3.94[18] \\
4.05[14]\end{array}$ & $\mathrm{T} 1$ \\
\hline 0.5 & $\begin{array}{l}4.046 \\
4.07[14]\end{array}$ & $\mathrm{O} 1, \mathrm{O} 2$ & $\begin{array}{c}4.098 \\
3.98[18] \\
4.11 ; 4.12 ; 4.14 \\
{[14]}\end{array}$ & $\mathrm{T} 1, \mathrm{~T} 4$ \\
\hline 0.75 & $\begin{array}{l}4.092 \\
4.11[14]\end{array}$ & $\mathrm{O} 1, \mathrm{O} 2, \mathrm{O} 3$ & $\begin{array}{c}4.168 \\
4.03[18] \\
4.18-4.20[14]\end{array}$ & $\mathrm{T} 1, \mathrm{~T} 4, \mathrm{~T} 7$ \\
\hline 1.0 & $\begin{array}{l}4.132 \\
4.15[14] \\
4.18[9] \\
4.13[7]\end{array}$ & $\mathrm{O} 1, \mathrm{O} 2, \mathrm{O} 3, \mathrm{O} 4$ & $\begin{array}{c}4.237 \\
4.07[18] \\
4.23 ; 4.24 ; 4.26 ; 4.27[14]\end{array}$ & $\mathrm{T} 2, \mathrm{~T} 3, \mathrm{~T} 5, \mathrm{~T} 8$ \\
\hline 1.25 & - & - & 4.297 & $\mathrm{~T} 1, \mathrm{~T} 2, \mathrm{~T} 3, \mathrm{~T} 5, \mathrm{~T} 8$ \\
\hline 1.5 & - & - & 4.355 & $\mathrm{~T} 1, \mathrm{~T} 2, \mathrm{~T} 3, \mathrm{~T} 4, \mathrm{~T} 6, \mathrm{~T} 7$ \\
\hline 1.75 & - & - & 4.410 & $\mathrm{~T} 1-\mathrm{T} 7$ \\
\hline 2.0 & - & - & 4.462 & $\mathrm{~T} 1-\mathrm{T} 8$ \\
\hline
\end{tabular}

ческой координации атомов водорода, что согласуется с работами других авторов $[1,4,11,12,22,25]$, в которых

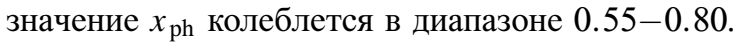

На рис. 3 представлены значения удельной энергии связи водорода в системе $\mathrm{PdH}_{x}$, рассчитанные по формуле

$$
E_{b}=\frac{\frac{N}{2} E_{\mathrm{H}_{2}}+E_{\mathrm{Pd}_{4}}-E_{\mathrm{Pd}_{4} \mathrm{H}_{N}}}{N},
$$

где $E_{\mathrm{H}_{2}}-$ полная энергия молекулы водорода, $E_{\mathrm{Pd}_{4}}-$ полная энергия, приходящаяся на 4 атома чистого палладия, $E_{\mathrm{Pd}_{4} \mathrm{H}_{N}}-$ полная энергия, приходящаяся на расчетную ячейку из 4 атомов чистого палладия и $N$ атомов водорода (рис. 1).

Как видно из рис. 3 , в отличии от параметра решетки и избыточного объема энергия связи водорода ведет себя нерегулярным образом. На начальном этапе энергия связи растет, достигает максимума при концентрации водорода $\sim 0.75$, а затем наблюдается ее уменьшение.

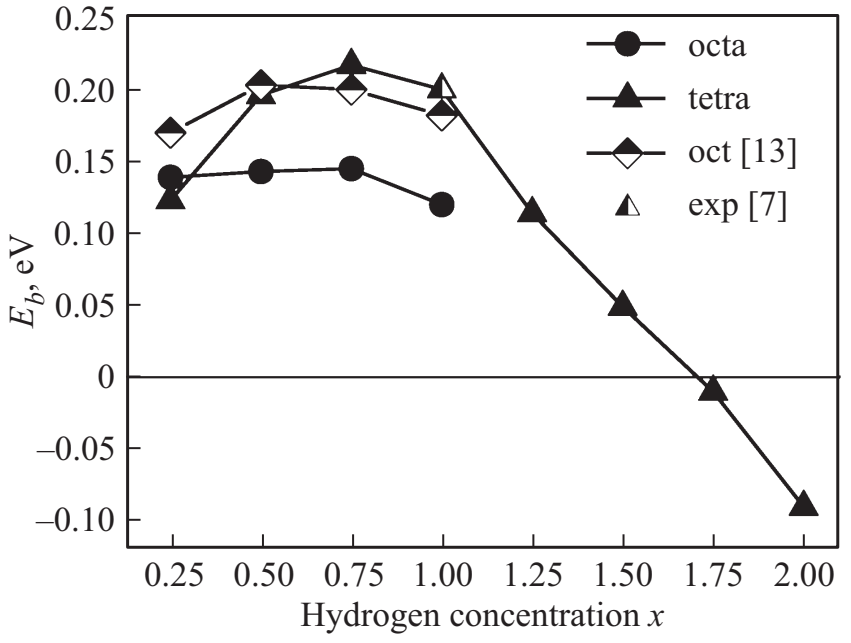

Рис. 3. Энергия связи водорода, рассчитанная на один атом $\mathrm{H}$, в зависимости от концентрации $x$ в палладии. 


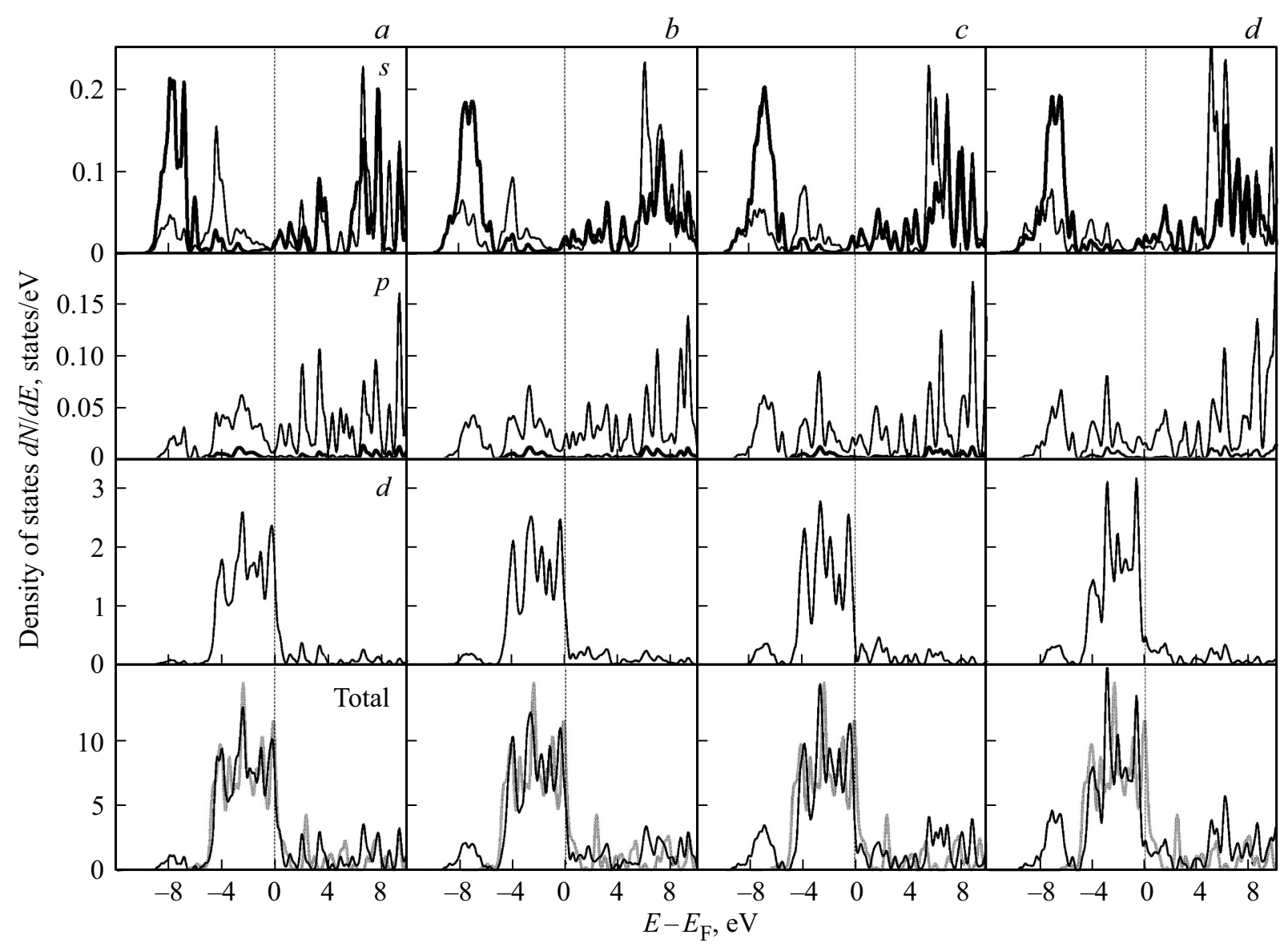

Рис. 4. Плотность электронных состояний (ПЭС) соединения $\mathrm{PdH}_{x}$ с октаэдрической координацией атома Н при $x$ равном $0.25(a), 0.5(b), 0.75(c)$ и $1.0(d)$. В трех верхних панелях показаны локальные парциальные плотности состояний $s$-, $p$ - и $d$-типа в МТ-сферах атомов Н (жирная черная линия) и атомов Рd (тонкая черная линия). В нижней панели показана полная ПЭС соединения $\mathrm{PdH}_{x}$ (тонкая черная линия) и чистого палладия (тонкая серая линия) в расчетной ячейке. Пунктирная линия уровень Ферми.

Отметим, что при концентрациях ниже $x=0.3$ энергетически выгодно положение водорода в О-междоузлиях, а выше - в Т-междоузлиях. Вместе с тем, согласно экспериментальным данным водород находится в октаэдрических междоузлиях. Расхождение с экспериментом обусловлено тем, что в нашей работе не рассматривались энергии нулевых колебаний атомов водорода в междоузлиях решетки палладия, учет которых приводит к согласию с экспериментальными данными $[1,2,13,14]$. При концентрациях водорода $x$ более 1.5 его энергия связи в палладии принимает отрицательные значения, что свидетельствует о неустойчивости таких соединений.

\section{4. Концентрационная зависимость электронной структуры системы $\mathrm{PdH}_{x}$}

\section{1. Плотность электронных состояний}

Рассчитанные в работе полные и локальные парциальные плотности электронных состояний (ПЭС) чи- стого $\mathrm{Pd}$ и $\mathrm{PdH}_{x}$ с октаэдрической и тетраэдрической координациями атомов Н в ГЦК-решетке металла, представлены на рисунках 4-6. Результаты, полученные для полных ПЭС чистого металла и $\mathrm{PdH}_{x}$ с октаэдрической координацией атомов $\mathrm{H}$ при $x$ равной $0.25,0.5$ и 1.0 хорошо согласуются с работами $[1,10,18,22,39,40]$.

В процессе растворения водорода в палладии наблюдаются следующие закономерности в поведении ПЭС:

1. С ростом концентрации водорода в решетке металла как в октаэдрическом, так и в тетраэдрическом междоузлиях ПЭС на уровне Ферми понижается в диапазоне $x=0-0.75$ ( $\alpha$-фаза), затем в диапазоне $x>0.75$ $(\beta$-фаза) для тетраэдрической координации - растет, для октаэдрической - выходит на плато (рис. 7). Таким образом, переход системы $\mathrm{PdH}_{x}$ из $\alpha$-фазы в $\beta$-фазу сопровождается заметным изменением электронных свойств [1]. Такое поведение ПЭС на уровне Ферми, определяющее проводимость системы, частично объясняет нерегулярное поведение электрического сопротивления $R$ в процессе растворения водорода в 


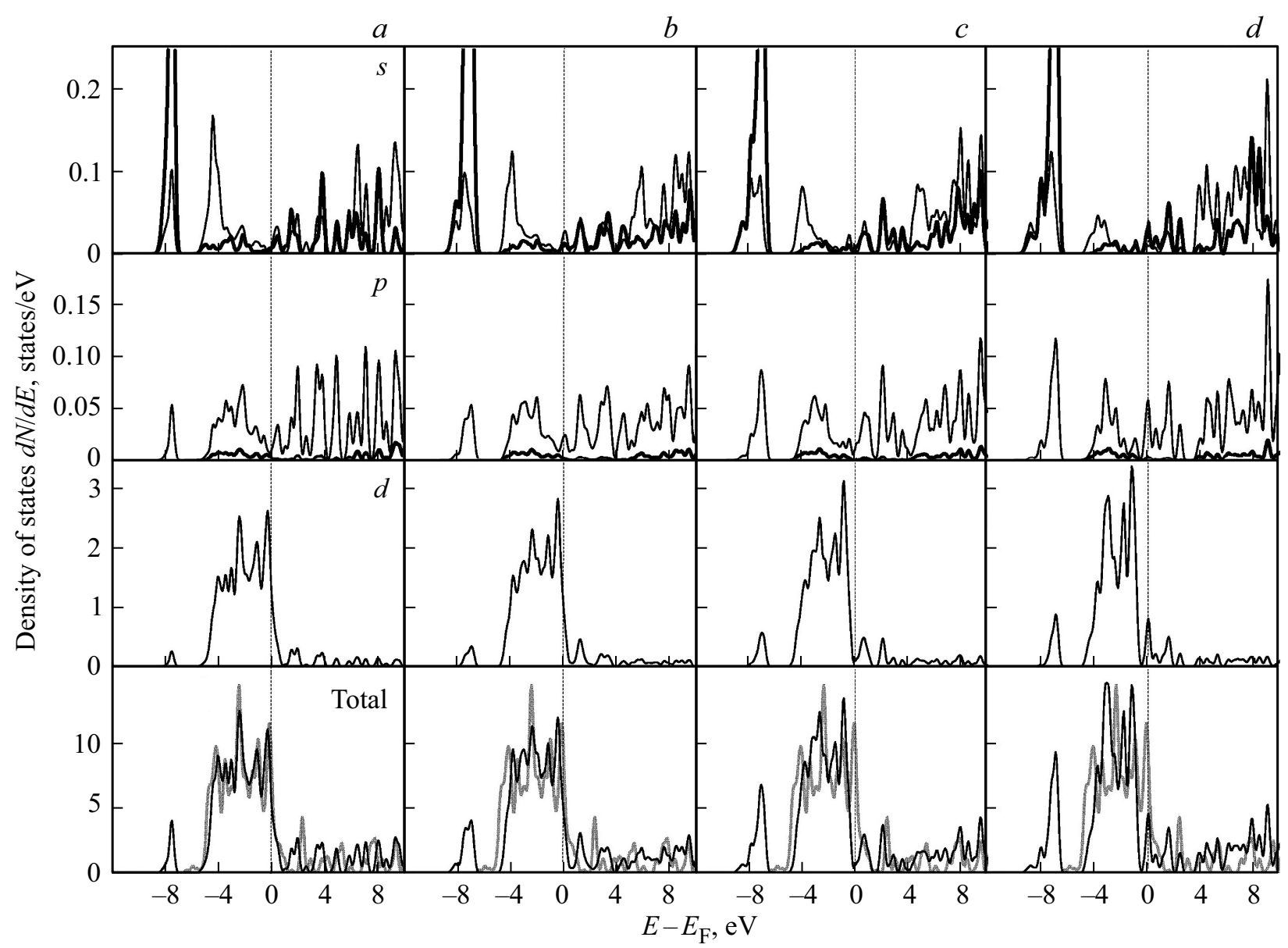

Рис. 5. ПЭС-соединения $\mathrm{PdH}_{x}$ с тетраэдрической координацией атома Н при $x$ равном $0.25(a), 0.5(b), 0.75(c)$ и $1.0(d)$. Использованы те же обозначения, что и на рис. 4.

палладии: рост $R$ вплоть до $x=0.75$, затем резкое падение [23-25]. При $x>1.0$ плотность электронных состояний на уровне Ферми снова начинает уменьшаться с ростом концентрации водорода.

2. Ниже дна валентной зоны Pd в процессе растворения водорода в ПЭС появляется пик, отделенный энергетической щелью. Чтобы понять природу состояний, формирующих этот пик, в работе были рассчитан ПЭС локальных парциальных $s$-, $p$ - и $d$-состояний соединений $\mathrm{PdH}_{x}$. Из анализа локальных парциальных ПЭС следует, что при насыщении водородом его $s$-состояния заполняются электронами $s$-, $p$ - и $d$-зон атомов металла, формируя гибридизованные металл-водородные состояния в энергетическом диапазоне от -6.5 до $-9.5 \mathrm{eV}$ при октаэдрической координации и от -7 до $-9 \mathrm{eV}$ при тетраэдрической координации атомов Н. Эти состояния обеспечивают химическую связь водорода с палладием. С ростом концентрации $x$ водорода ширина зоны этих состояний увеличивается нелинейно (рис. 8): при $x>0.5$ ширина этой зоны растет заметно медленнее, чем при $x<0.5$. При откаэдрической координации водорода ширина зоны гибридизованных $\mathrm{H}-\mathrm{Pd}$ состояний больше, чем при тетраэдрической, что, по-видимому, обусловлено большим расстоянием от атомов водорода в О-междоузлиях до атомов палладия.

3. Плотность электронных состояний в энергетической области $(6-10) \mathrm{eV}$ выше энергии ферми в соединениях $\mathrm{PdH}_{x}$ существенным образом отличаются от чистого Pd, как по количеству возбуждаемых состояний, так и по их интенсивности. При этом интенсивность возбуждаемых электронных состояний растет с концентрацией водорода. Наибольший вклад в эти состояния вносят $s$-состояния водорода (рис. 4-6), которые совместно с $s$-, $p$ - и $d$-состояниями Рd обеспечивают связь водорода с палладием. Возбуждение этих состояний, наиболее вероятно снижает связь водорода с палладием и тем самым облегчает его выход из металла. Данное предположение подтверждается экспериментальными результатами по исследованию стимулированного выхода водорода из Рd под действием ионизирующего излучения [26-28]. Облучение электронами палладия, предварительно насыщенного водородом или дейтерием, вызывает интенсивный выход изотопов водорода при комнатной и ниже температуре. Причем, скорость выхода водорода возрастает с ростом концентрации Н в Pd. 


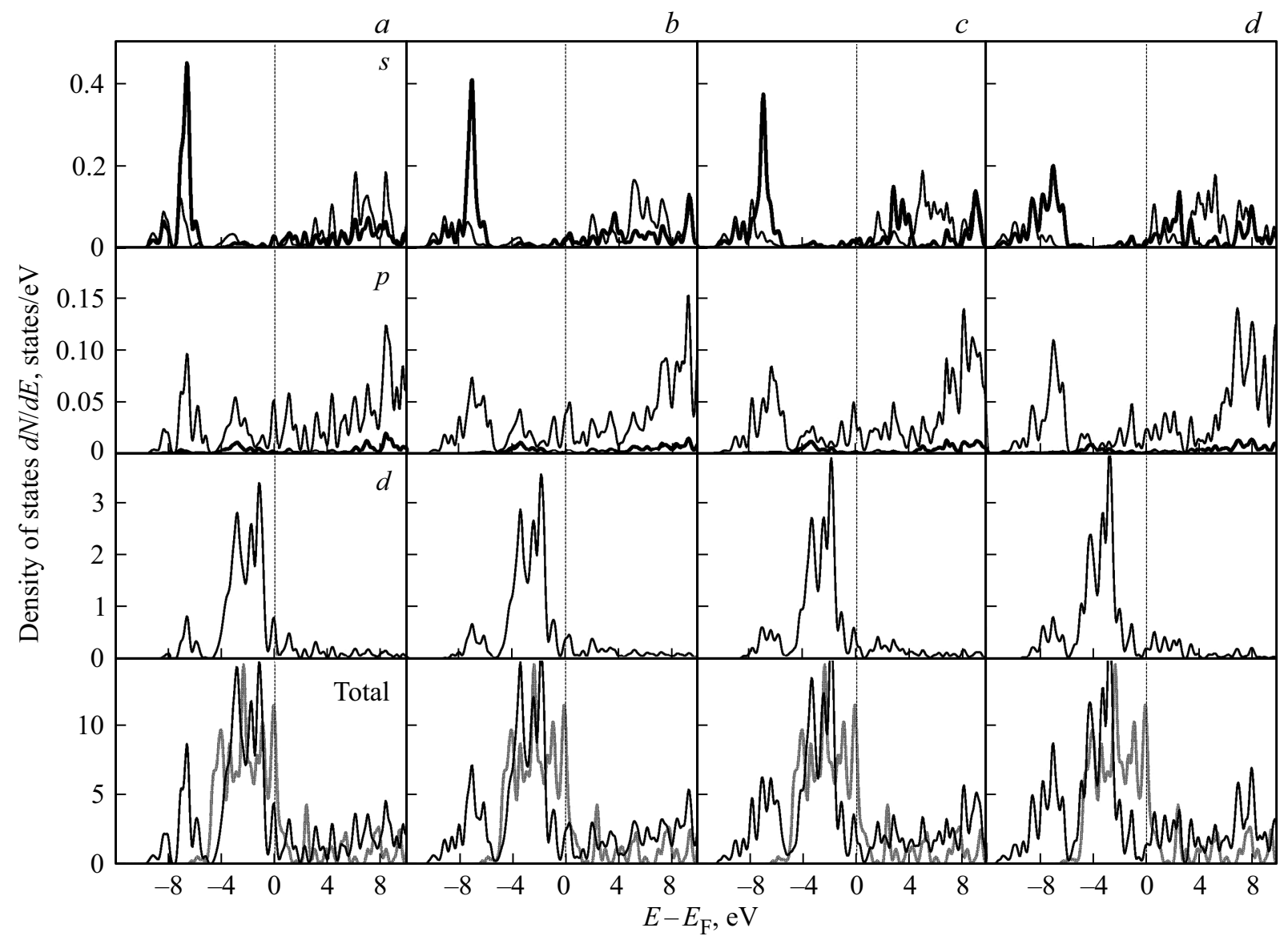

Рис. 6. ПЭС-соединения $\mathrm{PdH}_{x}$ с тетраэдрической координацией атома Н при $x$ равном $1.25(a), 1.5(b), 1.75(c), 2.0(d)$. Использованы те же обозначения, что и на рис. 4.

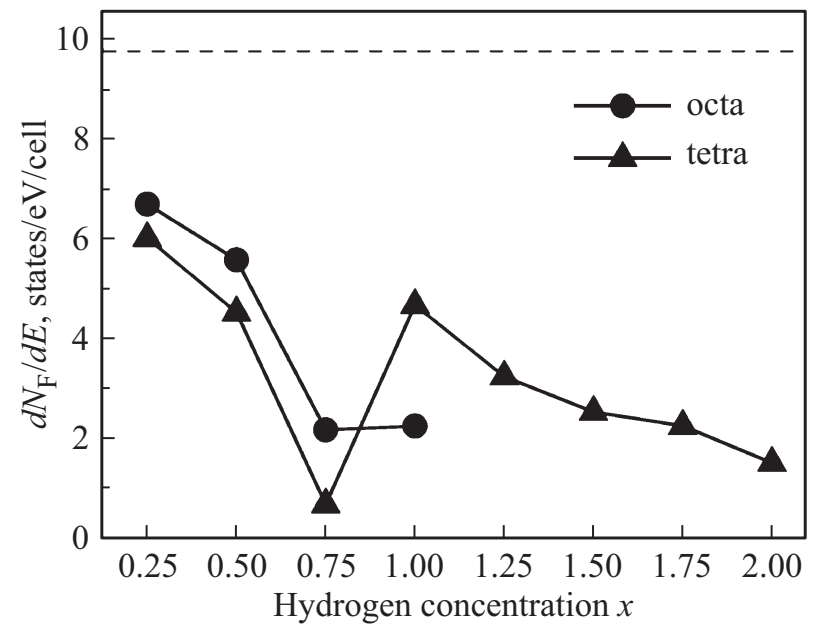

Рис. 7. Зависимость ПЭС на уровне Ферми соединения $\mathrm{PdH}_{x}$ от концентрации $x$ водорода. Пунктирная линия соответствует ПЭС на уровне Ферми чистого палладия.

\section{2. Перенос заряда}

В работе исследован перенос заряда между атомами водорода и палладия. С этой целью были рассчитаны по методу Бадера объемы, занимаемые атомами $\mathrm{Pd}$ в чистом металле, атомами $\mathrm{H}$ и $\mathrm{Pd}$ в соединении $\mathrm{PdH}_{x}$, зарядовые плотности и электронные заряды в этих объемах. На рис. 9 представлены концентрационные зависимости переноса заряда на атомах Н и $\mathrm{Pd}$ и объема

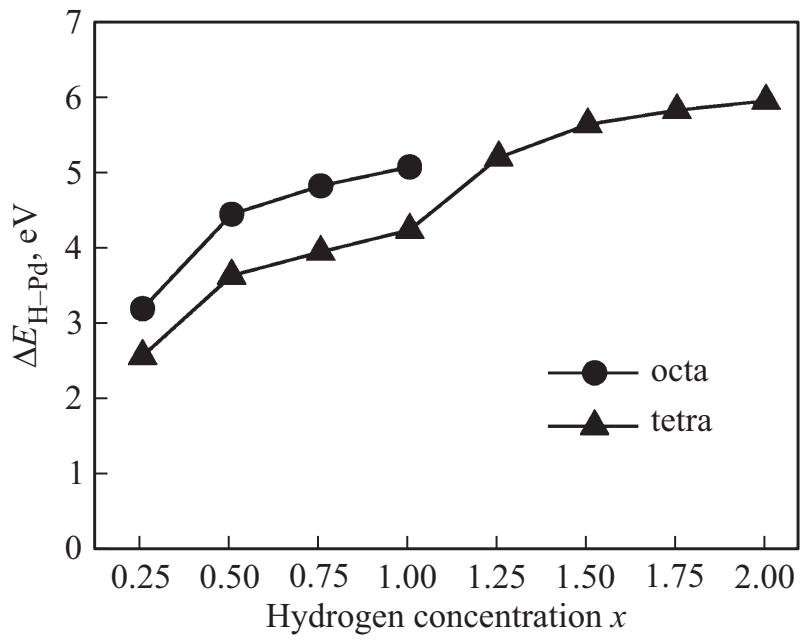

Рис. 8. Зависимость ширины зоны гибридизованных металлводородных состояний от концентрации $x$ водорода в палладии 

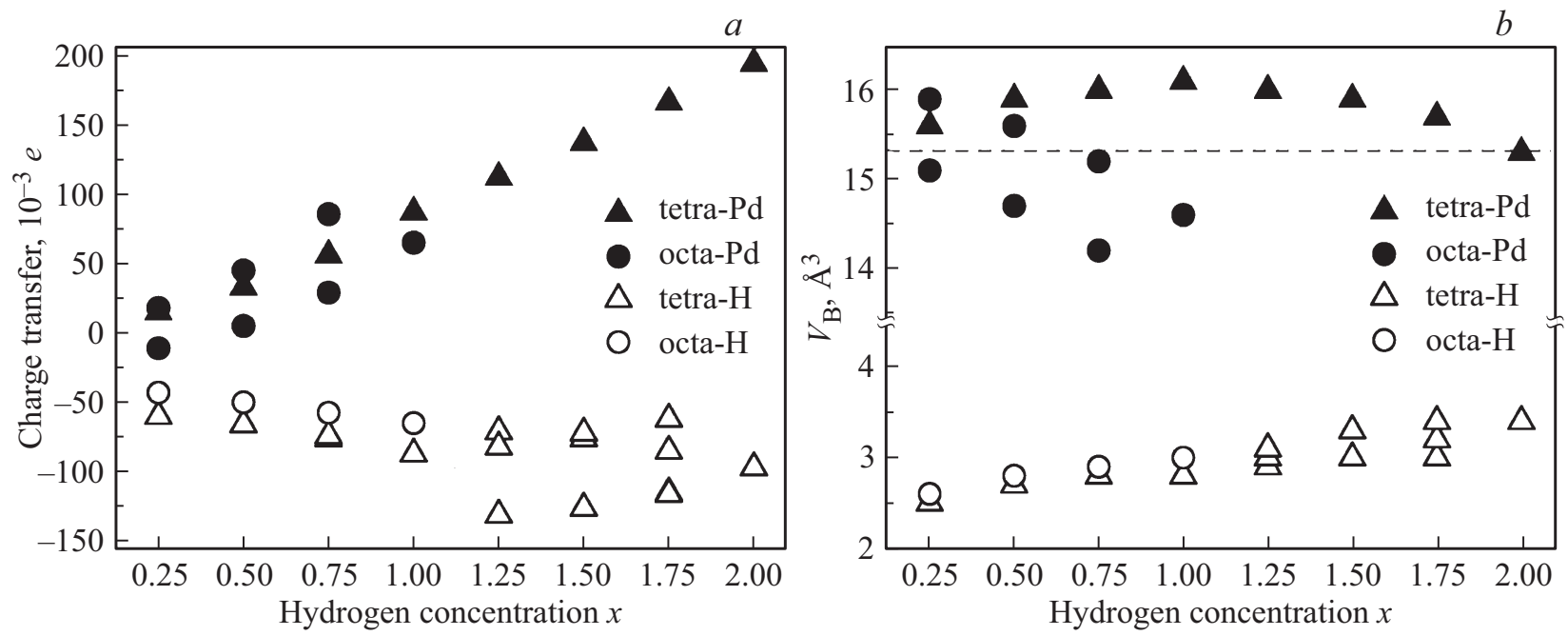

Рис. 9. Перенос заряда на атомах Н и Рd и объем атомов по Бадеру в зависимости от концентрации $x$ водорода в палладии. Пунктирная линия на панели $(b)$ соответствует объему атомов чистого палладия.

атомов по Бадеру. Независимо от координации атомов водорода перенос электронного заряда осуществляется от палладия к водороду, с ростом концентрации перенос заряда увеличивается (рис. 9, $a$ ). При линейной аппроксимации этих зависимостей угол ее наклона для атомов палладия больше, чем для атомов водорода во всем диапазоне концентрации водорода, то есть электронный заряд на атомах металла убывает быстрее, чем растет на атомах водорода. В следствие этого силы кулоновского отталкивания между атомами $\mathrm{Pd}$ с концентрацией $x$ растут быстрее, чем силы кулоновского притяжения между атомами водорода и палладия. Что, по-видимому, и приводит к уменьшению энергии связи водорода в металле при концентрациях $x>0.75$ : энергия кулоновского взаимодействия между одноименно заряженными атомами палладия начинает компенсировать энергию притяжения между атомами $\mathrm{H}$ и $\mathrm{Pd}$, ослабляя связь водорода в металле.

Перенос электронного заряда от Рd к Н в октаэдрических междоузлиях меньше, чем в тетраэдрических, что обусловлено, по-видимому, геометрическим фактором: большим расстоянием от атома $\mathrm{H}$ до атомов $\mathrm{Pd}$ в октаэдрических междоузлиях. Кроме этого, перенос заряда зависит от заполняемости междоузлий водородом. В частности, чем больше октаэдрических междоузлий в окрестности $\mathrm{Pd}$ занято $\mathrm{H}$ при одной и той же концентрации $x$, тем больше электронного заряда уходит от Pd к атомам водорода. При концентрациях $x>1$, на разных атомах водорода наблюдается разный перенос заряда. Совместный анализ рис. 1 и рис. 9, $a$ показал, что перенос заряда к атому водорода увеличивается при уменьшении заполняемости соседних междоузлий водородом. Перечисленные особенности переноса заряда в системе $\mathrm{PdH}_{x}$ свидетельствуют о зависимости силы взаимодействия между водородом и палладием от рас- положения атомов Н как относительно атома $\mathrm{Pd}$, так и относительно друг друга.

На рис. 9, $b$ представлена концентрационная зависимость объемов атомов Н и $\mathrm{Pd}$ по Бадеру. Объем атома водорода в октаэдрических междоузлиях больше, чем в тетраэдрических на величину, не превышающую $5 \%$, что связано с размерами пустот: октаэдрические превышают тетраэдрические междоузлия в 4 раза. С ростом $x$ объем атомов Pd при октаэдрической координации водорода уменьшается. Этот эффект усиливается с увеличением занятых водородом пустот в окрестности Рd. В пределе, когда атомы водорода занимают все октаэдрические междоузлия, объем, приходящий на каждый атом палладия, становится на $4.5 \%$ меньше, чем в чистом металле. В случае тетраэдрической координации водорода объем атомов Рd растет до концентрации $x=1.0$, затем спадает. Этот рост, по-видимому, обусловлен высоким удельным избыточным объемом, вносимым атомами водорода при растворении в палладии (рис. 2) при низких концентрациях.

Таким образом, в результате переноса заряда объемы, занимаемые атомами $\mathrm{H}$, становятся заряженными отрицательно, а атомами палладия - положительно. Перенос электронного заряд от атомов Рd растет по мере насыщения палладия водородом. В связи с этим возрастают кулоновские силы отталкивания заряженных атомов $\mathrm{Pd}$, что приводит к росту локальных напряжений в решетке и, как следствие, к водородному охрупчиванию металла.

\section{5. Заключение}

Проведены первопринципные расчеты атомной и электронной структур системы палладий-водород при концентрации водорода $x=\mathrm{H} / \mathrm{Pd}$ в диапазоне 0.25-2.0. 
В работе впервые изучен перенос заряда в системе палладий-водород в зависимости от концентрации водорода и его координации в решетке палладия.

Получено, что при заполнении водородом октаэдрических и тетраэдрических междоузлий решетка палладия остается ГЦК, при одновременном заполнении всех междоузлий наблюдается тетрагональное искажение решетки. Фазовый переход в системе палладий-водород происходит при $x=0.57$ для октаэдрической и при $x=0.80$ для тетраэдрической координации атомов водорода.

Выявлено, что энергия связи водорода с ростом его концентрации в палладии увеличивается и достигает максимума при $x \sim 0.75$, затем уменьшается. При $x>0.3$ энергетически выгодно положение водорода в тетраэдрических междоузлиях, при $x<0.3-$ в октаэдрических междоузлиях. При концентрациях $x>1.5$ энергия связи водорода в палладии принимает отрицательные значения, что свидетельствует о неустойчивости таких соединений.

Установлено, что ПЭС с ростом концентрации водорода на уровне Ферми понижается в диапазонах $x$ от 0 до 0.75 и от 1.0 до 2.0, что указывает на уменьшение плотности электронов проводимости системы $\mathrm{PdH}_{x}$. При растворении водорода в палладии ниже дна валентной зоны Pd появляются отделенные энергетической щелью состояния, которые образуются в результате заполнения $s$-состояний водорода электронами $s$-, $p$ - и $d$-зон атомов металла и формированием гибридизованных металлводородных состояний. Ширина этих зон растет с концентрацией водорода нелинейно. В области энергий (6-10) eV выше энергии Ферми в системе $\mathrm{PdH}_{x}$ ПЭС существенно больше, чем в чистом $\mathrm{Pd}$. Наибольший вклад в эти состояния вносят $s$-состояния водорода, которые совместно с $s$-, $p$ - и $d$-состояниями Pd обеспечивают связь водорода с палладием.

Установлено, что растворение водорода в $\mathrm{Pd}$ сопровождается переносом заряда от атомов Pd к атомам $\mathrm{H}$, в результате чего атомы металла становятся положительно заряженными, атомы водорода - отрицательно, причем этот перенос заряд растет по мере насыщения палладия водородом. Выявлено, что перенос заряда в соединениях $\mathrm{PdH}_{x}$, а, следовательно, и сила взаимодействия между водородом и палладием, сильно зависит от расположения атомов Н как относительно атома $\mathrm{Pd}$, так и относительно друг друга.

\section{Благодарности}

Работа была выполнена в рамках программы повышения конкурентноспособности Томского политехнического университета.

\section{Конфликт интересов}

Авторы заявляют, что у них нет конфликта интересов.

\section{Список литературы}

[1] Shun Dekura, Hirokazu Kobayashi, Kohei Kusada, Hiroshi Kitagawa. Chem. Phys. Chem 20, 1158 (2019).

[2] Jesse D. Benck, Ariel Jackson, David Young, Daniel Rettenwander, Yet-Ming Chiang. Chem. Mater. 31, 4234 (2019).

[3] F.A. Lewis. The palladium-hydrogen system. Academic, N.Y. (1967). $178 \mathrm{p}$.

[4] Водород в металлах / Под ред. Г. Алефельда, И. Фёлькля. Мир, М. (1981). Т. 1. С. 475; Т.2. С. 430.

[5] П.В. Гельд, Р.А. Рябов, Л.П. Мохрачева. Водород и физические свойства металлов и сплавов. Наука, М. (1985). $232 \mathrm{c}$

[6] W.B. Pearson. A Handbook of lattice spacings of metals and alloys. Pergamon, N.Y. (1967). 2, 1044 p.

[7] W.M. Mueller, J.P. Blackledge, G.G. Libowitz. Metal Hydrides. Academic, N.Y. (1968). 804 p.

[8] R.B. McLellan, W.A. Oates. Acta Met. 21, 181 (1973).

[9] A.R. Williams, J. Kübler, C.D. Gelatt. Jr. Phys. Rev. B 19, 6094 (1979).

[10] O.B. Christensen, P.D. Ditlevsen, K.W. Jacobsen, P. Stoltze, O.H. Nielsen, J.K. Nørskov. Phys. Rev. B 40, 1993 (1989).

[11] A.C. Bento, F.C.G. Gandra, E.C. da Silva, H. Vargas, L.C.M. Miranda. Phys. Rev. B 45, 5031 (1992).

[12] G.A. Tsirlina, M.D. Levi, O.A. Petrii, D. Aurbach. Electrochim. Acta 46, 4141 (2001).

[13] Xuezhi Ke, Gert Jan Kramer. Phys. Rev. B 66, 184304 (2002).

[14] Riccarda Caputo, Ali Alavi. Mol. Phys. 101, 1781 (2003).

[15] Xuezhi Ke, Gert Jan Kramer, Ole Martin Luvvik. J. Phys.: Condens. Matter 16, 6267 (2004).

[16] V.M. Silkin, I.P. Chernov, M. Echenique, Yu.M. Koroteev, E.V. Chulkov. Phys. Rev. B. 76, 245105 (2007).

[17] Y.J. Li, S.E. Kulkova, Q.M. Hu, D.I. Bazhanov, D.S. Xu, Y.L. Hao, R. Yang. Phys. Rev. B 76, 064110 (2007).

[18] K.G. McLennan, E. Mac, A. Gray, J.F. Dobson. Phys. Rev. B 78, 014104 (2008).

[19] O.Yu. Vekilova, D.I. Bazhanov, S.I. Simak, I.A. Abrikosov. Phys. Rev. B 80, 024101 (2009).

[20] Н.А. Кулабухова, Г.М. Полетаев, М.Д. Старостенков, П.Д. Голубь. Изв. АлтГУ. Сер. Математика и механика. Управление, вычислительная техника и информатика. Физика. 69, 160 (2011).

[21] Henrik Grönbeck, V.P. Zhdanov. Phys. Rev. B 84, 052301 (2011).

[22] M. Kofu, N. Hashimoto, H. Akiba, H. Kobayashi, H. Kitagawa, M. Tyagi, A. Faraone, J.R.D. Copley, W. Lohstroh, O. Yamamuro. Phys. Rev. B 94, 064303 (2016).

[23] G. Bambakidis, R.J. Smith, D.A. Otterson. Phys. Rev. 177, 1044 (1969).

[24] Y. Sakamoto, K Takai, I Takashima, M. Imada. J. Phys.: Condens. Matter 8, 3399 (1996).

[25] А.Ф. Вяткин, В.Т. Волков, В.Г. Еременко, Ю.А. Касумов, А.С. Колчина. Поверхность. Рентгеновские, синхротронные и нейтронные исследования 6, 35 (2015).

[26] Yu. Tyurin, I. Chernov. Int. J. Hydrogen Energy 27, 829 (2002).

[27] И.П. Чернов, Ю.М. Коротеев, В.М. Силкин, Ю.И. Тюрин. ДАН 420, 6, 1 (2008).

[28] Yu.I. Tyurin, V.S. Sypchenko, N.N. Nikitenkov, Hongru Zhang, I.P. Chernov. Int. J. Hydrogen energy 44, 20223 (2019). 
[29] D.R. Hamann. Phys. Rev. B 88, 085117 (2013).

[30] X. Gonze, B. Amadon, G. Antonius F. Arnardi, L. Baguet, J. Beuken, J. Bieder, F. Bottin, J. Bouchet, E. Bousquet, N. Brouwer, F. Bruneval, G. Brunin, T. Cavignac, J. Charraud, Wei Chen, M. Côté, S. Cottenier, J. Denier, G. Geneste, P. Ghosez, M. Giantomassi, Y. Gillet, O. Gingras, D.R. Hamann, G. Hautier, Xu He, N. Helbig, N. Holzwarth, Y. Jia, F. Jollet, W. Lafargue-Dit-Hauret, K. Lejaeghere, M.A.L. Marques, A. Martin, C. Martins, H.P.C. Miranda, F. Naccarato, K. Persson, G. Petretto, V. Planes, Y. Pouillon, S. Prokhorenko, F. Ricci, G. Rignanese, A.H. Romero, M.M. Schmitt, M. Torrent, M.J. van Setten, B. van Troeye, M.J. Verstraete, G. Zérah, J.W. Zwanziger. Comput. Phys. Commun. 248, 107042 (2020).

[31] A.H. Romero, D.C. Allan, B. Amadon, G. Antonius, T. Applencourt, L. Baguet, Jordan Bieder, F. Bottin, J. Bouchet, E. Bousquet, F. Bruneval, G. Brunin, D. Caliste, M. Côté, J. Denier, C. Dreyer, P. Ghosez, M. Giantomassi, Y. Gillet, O. Gingras, D.R. Hamann, G. Hautier, F. Jollet, G. Jomard, A. Martin, H.P.C. Miranda, F. Naccarato, G. Petretto, N.A. Pike, V. Planes, S. Prokhorenko, T. Rangel, F. Ricci, G. Rignanese, M. Royo, M. Stengel, M. Torrent, M.J. van Setten, B. van Troeye, M.J. Verstraete, J. Wiktor, J.W. Zwanziger, X. Gonze. J. Chem. Phys. 152, 124102 (2020).

[32] J.P. Perdew, K. Burke, E. Matthias. Phys. Rev. Lett. 77, 3865 (1996).

[33] C.G. Broyden. J. Institute Mathem. Its Appl. 6, 76 (1970).

[34] R. Fletcher. Comp. J. 13, 3, 317 (1970).

[35] D. Goldfarb. Math.Comput. 24, 109, 23 (1970).

[36] D.F. Shanno. Math. Comp. 24, 111, 647 (1970).

[37] R.F.W. Bader. Atoms in molecules. A Quantum Theory. Clarendon Press, Oxford (1990). 438 p.

[38] Ted B. Flanagan, W. A. Oates. Annu. Rev. Mater. Sci. 21, 269 (1991).

[39] C.T Chan, Steven G. Louie. Phys. Rev. 27, 6, 3325 (1983).

[40] Maiko Kofu, Naoki Hashimoto, Hiroshi Akiba, Hirokazu Kobayashi, Hiroshi Kitagawa, Kazuki Iida, Mitsutaka Nakamura, Osamu Yamamuro. Phys. Rev. B 96, 054304 (2017).

Редактор Т.Н. Василевская 